\title{
DNA Methylation Changes Induced by Redox-Active Compounds-Choosing the Right PCR-Based Method ${ }^{+}$
}

\author{
Patrycja Jakubek ${ }^{1, *}$, Jovana Rajić ${ }^{2}$, Monika Baranowska ${ }^{1}$, Melita Vidaković ${ }^{2}$, \\ Agnieszka Bartoszek ${ }^{1}$ and Jacek Namieśnik ${ }^{1}$ \\ 1 Faculty of Chemistry, Gdańsk University of Technology, 80-233 Gdańsk, Poland; \\ monika.baranowska@pg.edu.pl (M.B.); agnieszka.bartoszek@pg.edu.pl (A.B.); \\ jacek.namiesnik@pg.edu.pl (J.N.) \\ 2 Institute for Biological Research "Siniša Stanković", University of Belgrade, 11060 Belgrade, Serbia; \\ jovana.rajic@ibiss.bg.ac.rs (J.R.); melita@ibiss.bg.ac.rs (M.V.) \\ * Correspondence: patrycja.jakubek@pg.edu.pl \\ + Presented at Natural Products and the Hallmarks of Chronic Diseases-COST Action 16112, Luxemburg \\ 25-27 March 2019.
}

Published: 17 April 2019

\begin{abstract}
The impact of catechins on the expression profile of redox-related genes in HT29 cell line has been studied recently by our group using Oxidative Stress RT2 Profiler PCR Array. Within the examined panel of 84 genes, the down-regulation of SRXN1 gene was unique among other up-regulated genes. We hypothesized that the observed down-regulation resulted from DNA methylation and have exploited this observation to choose the proper strategy to monitor the changes in DNA methylation patterns incurred by dietary antioxidants. The current study verified two PCR-based approaches.
\end{abstract}

Keywords: catechins; DNA methylation; methylation-specific PCR; methylation-sensitive high resolution melting; redox homeostasis; epigenetics

\section{Introduction}

The role of phytochemicals in epigenetic processes, such as DNA methylation, has been of major interest over several years. The choice of an appropriate method for assessment of DNA methylation depends on the biological question posed. High throughput techniques find its application in a measurement of whole genome methylation status, whereas techniques of low throughput are used to evaluate methylation levels of a region of interest. The second group includes PCR-based methods: methylation-specific PCR (MSP) and methylation-sensitive high-resolution melting (MS-HRM), which differ by reproducibility, sensitivity and specificity. The initial step before any analysis is to transform DNA in a bisulfite conversion reaction in order to distinguish methylated cytosines from unmethylated ones within the investigated sequence. MSP is a simple method and to perform it, two pairs of primers are needed: one specific to methylated and the other to unmethylated $\mathrm{CpG}^{\prime} \mathrm{s}$. This approach requires two separate PCR reactions to be run for each sample. In MS-HRM method, the methylation level is based on the melting profile of a PCR product, which must be compared to standards of a known percentage of methylation. MSP method assesses methylation of C $\mathrm{PG}^{\prime}$ s within primer-binding sites only, while MS-HRM evaluates the ratio of methylated to unmethylated $\mathrm{CpG}^{\prime}$ s present within a sequence delineated with designed primer pair [1].

In a previous study, we reported that exposure of HT29 cell line to physiological concentration of catechins up-regulates the expression of genes related to oxidative stress defense [2]. On the other 
hand, the higher $-10 \mu \mathrm{M}$-concentration, relevant only for the intestinal cells which are in a direct contact with consumed food, seems to preserve cellular redox state. The expression of redox-related genes remained unaffected, with the exception of the down-regulation of SRXN1 gene by (-)-epigallocatechin (EGC). The SRXN1 gene encodes for sulfiredoxin (SRX)-the enzyme responsible for restoring the activity of hydrogen peroxide scavengers, namely peroxiredoxins (PRXS) that under oxidative stress conditions become oxidized and inactivated [3]. The increased expression of SRXN1 has been shown in various types of cancer, including colon cancer. However, it is not up-regulated in mouse or human normal colon epithelial cells or cancer adjacent normal tissues [ $4-6]$.

The objective of this study was to choose the proper methodology for the detection of changes in methylation profile of CPG islands within the promoter area of SRXN1 gene in HT29 cell line exposed to model dietary antioxidants. For the study, three compounds were chosen, that in previous experiments displayed different gene expression responses: two catechins and glutathione, which is not only produced endogenously but also present in many foods.

\section{Materials and Methods}

\subsection{Cell Culture Conditions and Treatment with Antioxidants}

Human colon adenocarcinoma cell line (HT29) was cultured in supplemented McCoy's medium in a humidified atmosphere with $5 \% \mathrm{CO}_{2}$ at $37^{\circ} \mathrm{C}$ as described previously [2]. Cells for DNA methylation analysis were seeded in 6-well tissue culture plates and treated with (+)-catechin (C), (-)-epigallocatechin (EGC) (Extrasynthese) or glutathione (GSH) (Sigma-Aldrich, St. Louis, MO, USA) for $24 \mathrm{~h}$ at $37^{\circ} \mathrm{C}$. The experiments were performed in three independent biological replicates and with adequate control cells (treated with $3 \%$ ethanol or water).

\subsection{Genomic DNA Isolation and Bisulfite Conversion}

Genomic DNA was isolated from treated and control cells using QuickDNA Miniprep Plus Kit from Zymo Research (Irvine, CA, USA). Subsequently, isolated genomic DNA underwent bisulfite conversion with EZ DNA Methylation kit (Zymo Research, Irvine, CA, USA).

\subsection{Prediction of $C p G$ Islands and Design of Primers}

CPG island of human SRXN1 gene (SRXN1; NC_000020.11) was predicted with EMBOSS CpGplot using standard parameters. The sequence of genomic DNA uploaded for the analysis comprised the region of $1000 \mathrm{bp}$ upstream and $395 \mathrm{bp}$ downstream. Methylated (M) and unmethylated (U) sets of primers for MSP and MS-HRM analyses were designed using MethPrimer. The sequences of the used primers (Invitrogen, Waltham, MA, USA): forward M1 5'-GTTAGATTGGAAGTGGAATCGTT-3', reverse M1 5'-CCAAAATAAATCGACAAAACCC-3', forward U1 5'-GTTAGATTGGAAGTGGAATTGT-3', reverse U1 5'-AACCAAAATAAATCAACAA AACCC-3'.

\subsection{Methylation-Specific PCR}

For MSP, Maxima SYBR Green/ ROX qPCR Master Mix (2X) (Thermo Scientific, Waltham, MA, USA) was used. Thermal cycling conditions of MSP were as follows: initial denaturation $\left(95^{\circ} \mathrm{C}, 10\right.$ $\mathrm{min})$, then 40 cycles of 3-step protocol: denaturation $\left(95^{\circ} \mathrm{C}, 15 \mathrm{~s}\right)$, annealing $\left(57^{\circ} \mathrm{C}, 30 \mathrm{~s}\right)$ and extension $\left(72{ }^{\circ} \mathrm{C}, 30 \mathrm{~s}\right)$. Specificity of PCR products was verified with the melting curve analysis performed at the following thermal conditions: $95^{\circ} \mathrm{C}$ for $15 \mathrm{~s}, 60^{\circ} \mathrm{C}$ for $60 \mathrm{~s}$ and $95{ }^{\circ} \mathrm{C}$ for $1 \mathrm{~s}$. MSP was performed in QuantStudio 3 Real-Time PCR System from Applied Biosystems (Waltham, MA, USA). Relative DNA methylation was calculated based on the difference of $C_{t}$ values for methylated and unmethylated PCR products. 


\subsection{Methylation-Sensitive High Resolution Melting}

For the identification of all possible variants in DNA methylation status, each reaction contained a mix of all 4 primers (methylated and unmethylated pairs). PCR amplification and subsequent HRM analysis were performed in QuantStudio 3 Real-Time PCR System from Applied Biosystems (Waltham, MA, USA). The thermal cycling conditions included initial denaturation step $\left(95^{\circ} \mathrm{C}, 10 \mathrm{~min}\right.$ ) with subsequent 3-step PCR consisting of 40 cycles of denaturation $\left(95{ }^{\circ} \mathrm{C}, 15 \mathrm{~s}\right.$ ), annealing $\left(59{ }^{\circ} \mathrm{C}, 30 \mathrm{~s}\right)$ and elongation $\left(72{ }^{\circ} \mathrm{C}, 30 \mathrm{~s}\right)$. The protocol was finished with $7 \mathrm{~min}$ of subsequent final extension at $72{ }^{\circ} \mathrm{C}$. PCR amplification was followed by HRM that consisted of denaturation $\left(95^{\circ} \mathrm{C}, 15 \mathrm{~s}\right)$, annealing $\left(60^{\circ} \mathrm{C}, 60 \mathrm{~s}\right)$ and the final denaturation step $\left(95^{\circ} \mathrm{C}, 15 \mathrm{~s}\right)$ with a ramp of $0.025^{\circ} \mathrm{C}$ per second. For the end-product analysis, HRM Software c3.1 (Applied Biosystems, Waltham, MA, USA) was applied. Standard curves for MS HRM analysis were prepared using human methylated and non-methylated DNA standards (Zymo Research, Irvine, CA, USA), which first underwent bisulfite conversion reaction as described at the point 2.2. Subsequently, the converted methylated and non-methylated DNA samples were mixed to obtain standards of 0,50 and $100 \%$ methylation. After normalization of obtained results, the linear regression equation was used to quantitatively calculate the percentage of methylation of each sample.

\section{Results}

The SRXN1 gene is $6.7 \mathrm{~kb}$ long with the promoter area of $2.7 \mathrm{~kb}$ according to GeneCards (Human Gene Database). Using the EMBOSS CpGplot online tool, we identified one CpG island of 706 bp length located at -366 to +340 from the transcription starting site (TSS) and designed sets of methylated and unmethylated primers (M1/U1) that bind within this sequence as shown in Figure 1.

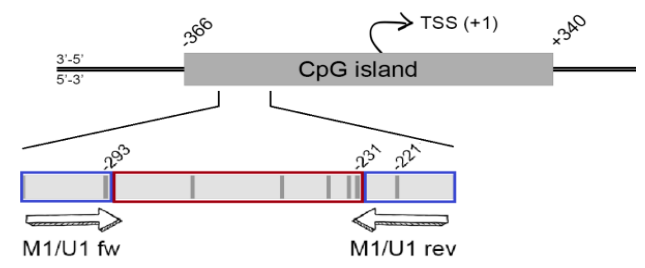

Figure 1. Schematic presentation of promoter area of the SRXN1 gene encompassing investigated $\mathrm{CpG}$ island. Primer binding sites are shown in the inset with $\mathrm{CpG}$ dinucleotides marked as short vertical lines. The TSS location is marked as " 1 "; M1/U1 fw, methylated and unmethylated forward primers, respectively; M1/U1 rev, methylated and unmethylated reverse primers, respectively. Blue outlining indicates regions investigated with MSP, whereas red one-MS-HRM.

Methylation status of specific CpG's investigated with MSP method did not differ between control and investigated compounds (C, EGC, GSH at $10 \mu \mathrm{M}$ concentration). On the other hand, MS-HRM analysis showed significant changes in the methylation level within the region of interest, but only after treatment with catechins. The treatment of HT29 cells with glutathione did not influence DNA methylation or expression of SRXN1 gene. As hypothesized, $10 \mu \mathrm{M}$ EGC that was reported to down-regulate the expression of SRXN1 gene, was also able to induce significant increase in the DNA methylation level within the CpG island of interest. However, the impact of $(+)$-catechin was opposite to EGC, i.e., in this case DNA methylation was significantly decreased.

\section{Discussion}

Based on the results of microarray analysis from the previous study [2], we expected that DNA of cells treated with EGC at $10 \mu \mathrm{M}$ concentration would be more methylated within the examined CPG island of SRXN1 promoter, since such a treatment caused down-regulation of this gene. These initial assumptions were confirmed only in the experiments carried out by MS-HRM approach. In the case of (+)-catechin (parent structure of EGC) and glutathione (of similar redox potential to EGC standard), no change in this gene methylation profile was detected by any of two PCR-based 
methods tested. Such a result was expected, because these two compounds did not alter SRNX1 gene expression.

MSP and MS-HRM methods, at least theoretically, reveal different insights into DNA methylation process in terms of different $\mathrm{CpG}^{\prime} \mathrm{s}$, but also differences in the occurrence of cytosines in the sequence analysed. In the case of our investigations, MSP analysis did not show any changes in the methylation status of specific cytosines in the sequence of interest. However, the difference was observed when the bigger area of CPG island was analysed by MS-HRM. The results obtained with this second approach showed that treatment of HT29 cells with $10 \mu \mathrm{M}$ EGC resulted in elevated DNA methylation level, which is consistent with down-regulated gene expression. Based on these observations, MS-HRM method seems more appropriate for the further investigation of changes in DNA methylation status in the promoter area of SRXN1 gene induced by phytochemicals exerting antioxidant activity.

Interestingly, the data provided additional insight into the possible mechanisms of catechin impact on DNA methylation. Until now, catechins have been reported only to act as inhibitors of DNA methyltransferases, which was reflected by the decrease in DNA methylation demonstrated for e.g., (+)-catechin. Current results demonstrate that these flavonoids may also be capable of increasing the level of methylated cytosines.

Author Contributions: Conceptualization, A.B.; methodology, M.V. and J.R.; formal analysis, P.J. and M.B.; investigation, P.J.; writing-original draft preparation, P.J.; writing-review and editing, M.V., A.B.; visualization, P.J., J.R.; supervision, A.B., M.V.; funding acquisition, J.N.

Funding: Project „Antioxidant Power Series as a tool rational design and assessment of health promoting properties of functional food based on antioxidant phytochemicals" (number of the application 2014/14/A/ST4/00640) financed by National Science Centre, Poland in a programme „MAESTRO 6". This study has been supported in part by the Ministry of Education, Science and Technological Development of the Republic of Serbia, Grant No. 173020.

Acknowledgments: This article is based upon work from COST Action NutRedOx-CA16112 supported by COST (European Cooperation in Science and Technology).

Conflicts of Interest: The authors declare no conflict of interest.

\section{References}

1. Akika. R.; Awada, Z.; Mogharbil, N.; Zgheib, N.K. Region of interest methylation analysis: A comparison of MSP with MS-HRM and direct BSP. Mol. Biol. Rep. 2017, 44, 295-305, doi:10.1007/s11033-017-4110-7.

2. Baranowska, M.; Suliborska, K.; Chrzanowski, W.; Kusznierewicz, B.; Namieśnik, J.; Bartoszek, A. The relationship between standard reduction potentials of catechins and biological activities involved in redox control. Redox Biol. 2018, 17, 355-366, doi:10.1016/j.redox.2018.05.005.

3. Hyun, A.W.; Jeong, W.; Chang, T.-S.; Park, K.J.; Park, S.J.; Yang, J.S.; Rhee, S.G. Reduction of cysteine sulfinic acid by sulfiredoxin is specific to 2-Cys peroxiredoxins. J. Biol. Chem. 2005, 280, 3125-3128, doi:10.1074/jbc.C400496200.

4. Wei, Q.; Jian, H.; Baker, A.; Dodge, L.K.; Gerard, M.; Young, M.R.; Toledano, M.B.; Colburn, N.H. Loss of sulfiredoxin renders mice resistant to azoxymethane/dextran sulfate sodiuminduced colon carcinogenesis. Carcinogenesis 2013, 34, 1403-1410, doi:10.1093/carcin/bgt059.

5. Wei, Q.; Jiang, H.; Matthews, C.P.; Colburn, N.H. Sulfiredoxin is an AP-1 target gene that is required for transformation and shows elevated expression in human skin malignancies. PNAS 2008, 105, 19738-19743, doi:10.1073/pnas.0810676105.

6. Wei, Q.; Jiang, H.; Xiao, Z.; Baker, A.; Young, M.R.; Veenstra, T.D.; Colburn, N.H. Sulfiredoxin-Peroxiredoxin IV axis promotes human lung cancer progression through modulation of specific phosphokinase signaling. PNAS 2011, 108, 7004-7009, doi:10.1073/pnas.1013012108. 